\title{
NEK2 induces osteoclast differentiation and bone destruction via heparanase in multiple myeloma
}

Leukemia (2017) 31, 1648-1650; doi:10.1038/leu.2017.115

To positively modulate multiple myeloma ( $\mathrm{MM}$ ) bone disease, understanding the mechanism of bone destruction is required to provide better targeted therapy. Specific molecular genetic lesions and tumor cell-stroma interactions influence the clinical course and response to therapy, all of which can vary markedly.,2 Our recent study demonstrates that an increased chromosomal instability (CIN) signature is associated with drug resistance and poor prognosis. ${ }^{3,4}$ High expression of NEK2, one of the CIN genes, has been shown to induce premature splitting of centrosomes, while suppression of NEK2 blocks spindle and chromosome segregation, resulting in accumulation of multiple centrosomes., Interestingly, we found that NEK2 expression is highly correlated with the expression of Heparanase 1 (HPSE), and its expression is significantly increased in the few myeloma cells still present in complete remission and not unexpectedly also in relapse. ${ }^{3}$ Although the tumor cell number is markedly decreased in remission patients, bone osteolysis still continues, suggesting that drug-resistant MM cells with high

a

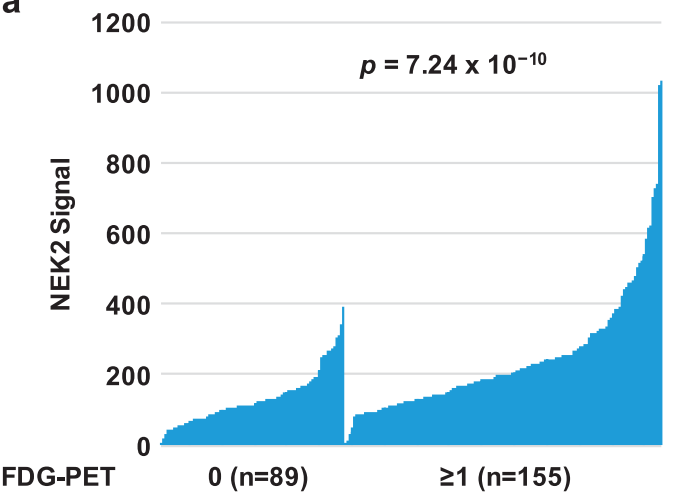

expression of NEK2 and HPSE, may play a critical role in bone destruction or prevent the repair of bone disease during remission.

In this study, we investigated whether MM patients with high expression of NEK2 in MM cells show increased focal bony lesions. NEK2 expression was examined in $\mathrm{CD}_{138^{+}} \mathrm{MM}$ cells using Affymetrix U133Plus2 microarray (Affymetrix, Santa Clara, CA, USA) and correlated with bone focal lesions determined by either fluorine-18 fluorodeoxyglucose positron emission tomography (FDG-PET) scan or magnetic resonance imaging (MRI) in 244 newly diagnosed MM patients enrolled in the total therapy 2 (TT2) clinical trial. ${ }^{7,8}$ We found that NEK2 levels were positively correlated with focal lesions as assessed by both FDG-PET and MRI. On FDG-PET test, 89 cases showed no detectable bone lytic lesion and 155 cases had one or more lesions. ${ }^{9,10}$ NEK2 levels were higher in purified MM cells from patients who had one or more lesions compared to patients with no lytic lesions on FDG-PET (the median signals of probe set 211080_s_at were 114.8 and 198.2, respectively, $P=7.24 \times 10^{-10}$ ) (Figure 1a). NEK2 expression was also compared between MM patients with or without bone lytic lesions defined by MRI. As shown in Figure 1b, NEK2 signals in patients with one or more focal bone lesions $(n=170)$ were

b
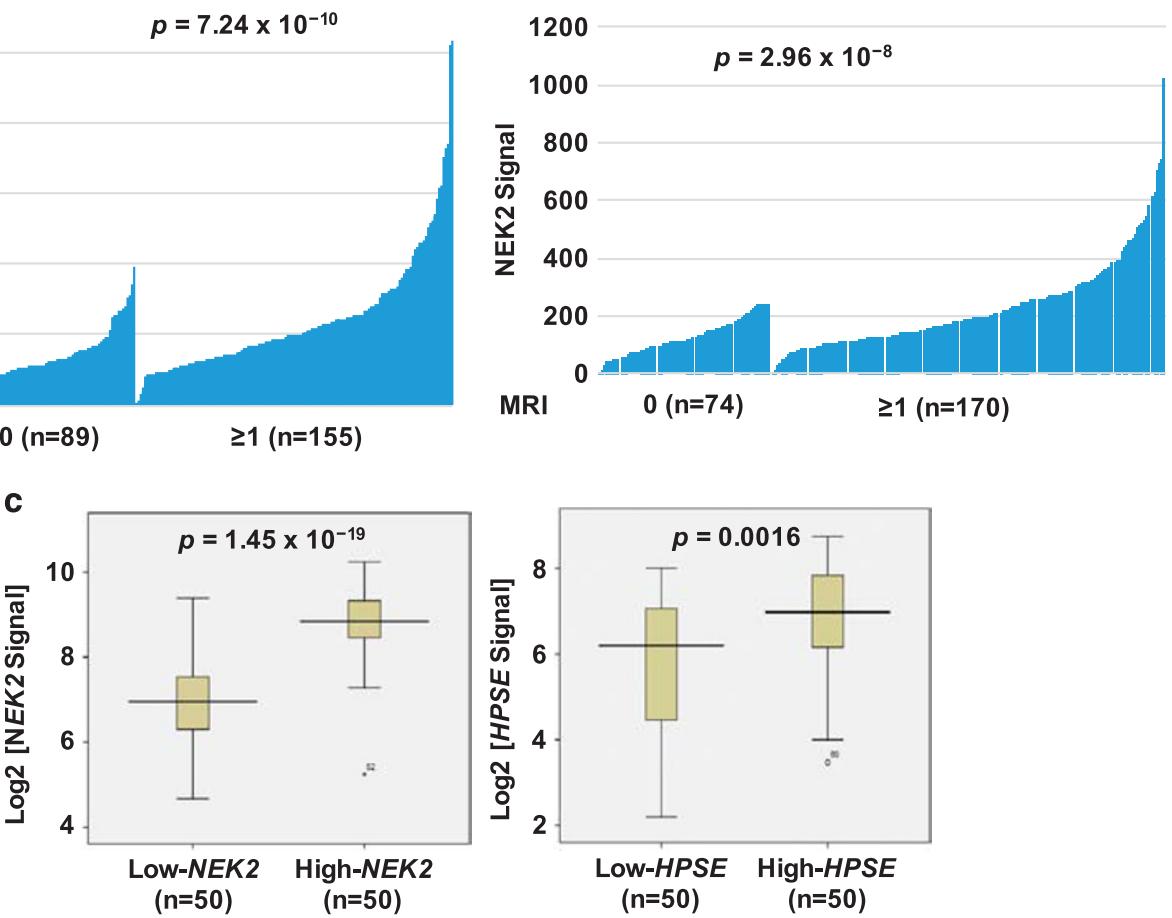

Figure 1. High expression of NEK2 in MM cells is positively correlated with bone lytic lesions in newly diagnosed MM patients. (a) Bar-view represents the correlation of NEK2 gene expression in MM cells from 89 patients without bone lytic lesion and 155 patients with one or more bone lesions on FDG-PET scan. (b) Bar-view represents the correlation of NEK2 gene expression in MM cells from 74 patients without bone lytic lesion and 174 patients with one or more bone lesions on MRI. (c) Box-plots show the expression of NEK2 and HPSE in 50 samples with high-NEK2 and low-NEK2. 


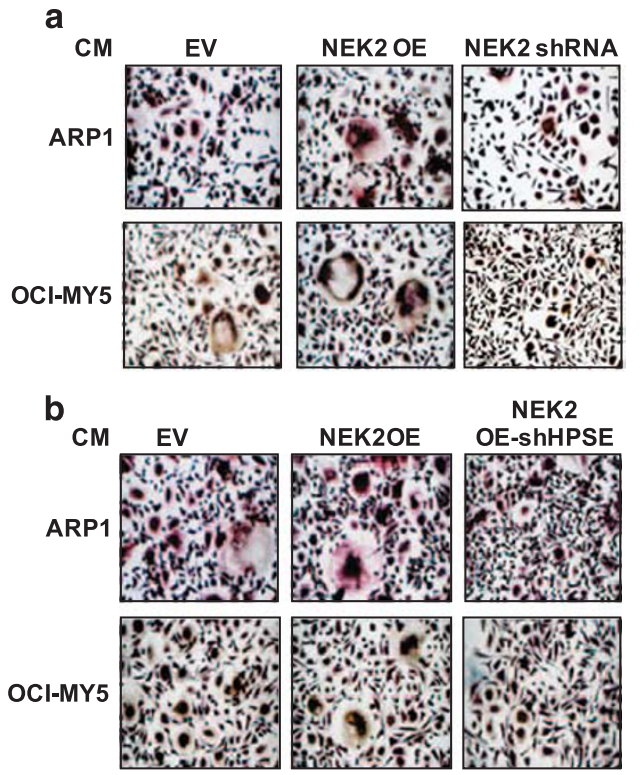

C
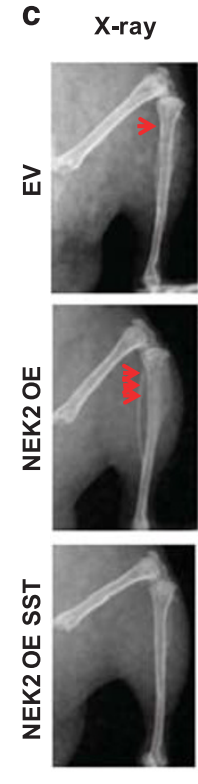

microct
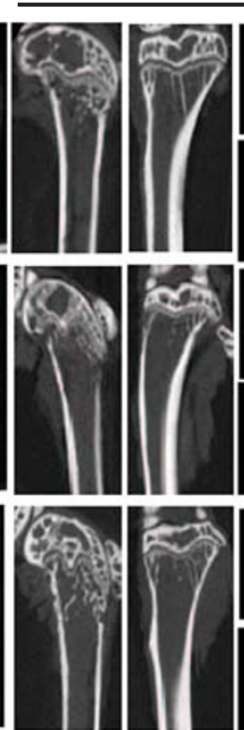

H\&E
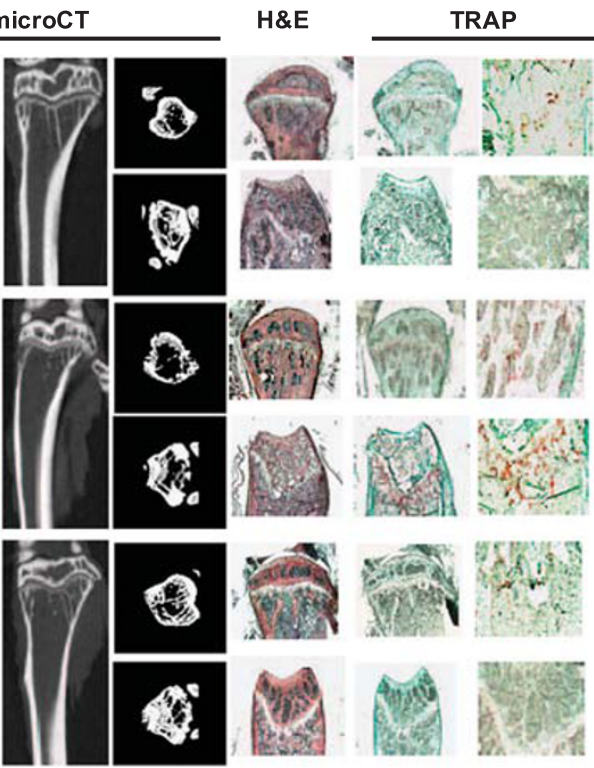

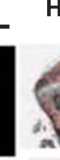
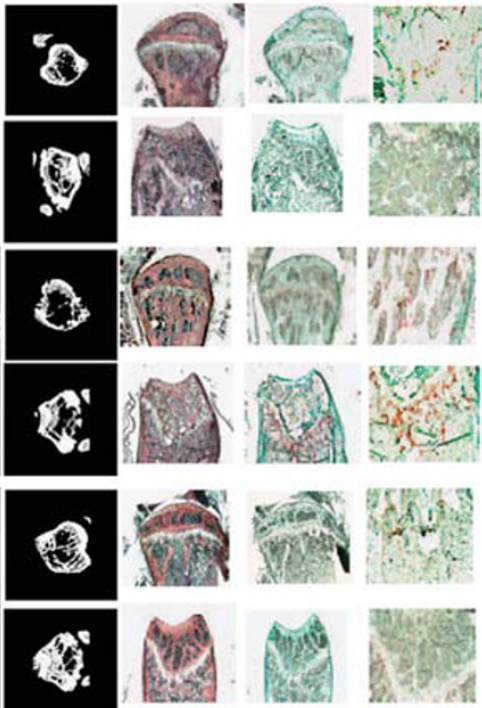

Figure 2. Overexpression of NEK2 in MM cells enhances osteoclast differentiation and bone destruction. (a) Human bone marrow macrophages (BMMs) from MM patients were cultured in the conditioned media collected from the control (EV), NEK2 OE and NEK2 shRNA ARP1 and OCl-MY5 lines for 14 days. Osteoclasts were detected by TRAP staining. (b) BMMs were cultured in the conditioned media collected from the control (EV), NEK2 OE, and NEK2 OE-shRNA HPSE (NEK2 OE shHPSE) ARP1 and OCI-MY5 lines for 14 days. Osteoclasts were detected by TRAP staining. (c) Representative X-ray, $\mu \mathrm{CT}$ and histological images for H\&E and TRAP staining of bones from mice treated with ARP1 EV, ARP1 NEK2 OE and NEK2 OE plus Roneparstat (NEK2 OE SST). Red arrows indicate bone lytic lesions by X-ray.

significantly higher compared to patients without bone lytic lesions ( $n=74$; the median signals were 189.8 and 115.6, respectively, $P=2.96 \times 10^{-8}$ ). These data suggest that high NEK2 expression in MM cells correlates indeed with focal bone lesions in MM patients.

In order to determine the factor related to NEK2 and causing bone damage in MM disease, we examined genes highly correlated with NEK2 expression in MM cells which had been published by our group previously. ${ }^{3}$ HPSE was one of the 70 genes highly positively correlated with NEK2 expression in MM cells. HPSE is an enzymatically active endoglycosidase that degrades heparan sulfate at specific intra-chain sites. ${ }^{11}$ Expression of HPSE is relatively low or undetectable in normal tissues but it is upregulated in many human tumors, including MM. It was recently demonstrated that heparanase promotes chemoresistance in MM. ${ }^{12}$ High expression of HPSE has also been linked to local and systemic osteolysis in $M M .{ }^{13}$ We compared HPSE expression in primary MM cells between 50 highNEK2 and 50 low-NEK2 samples, HPSE is highly positively correlated with NEK2 expression (Figure 1c). Analysis of GEPs in ARP1 MM cells, which either overexpressed NEK2 (NEK2 OE) or with silenced NEK2 (NEK2 shRNA) revealed that HPSE was highly correlated with NEK2 expression. GEP data showed that HPSE was increased 2.5 fold in NEK2 OE MM cells, and decreased 3.3-fold in NEK2-shRNA ARP1 cells compared to the control. ${ }^{3}$ We then verified this positive correlation between NEK2 and HPSE expression using both quantitative reverese transcription PCR and western blotting. HPSE expression was significantly upregulated in ARP1 and OCI-MY5 NEK2 OE MM cells at both the mRNA and protein levels (Supplementary Figures S1a and b) when compared to ARP1 and OCI-MY5 cells infected with empty vector (EV), while it was markedly downregulated in ARP1 and OCI-MY5 cells with silenced NEK2 (Supplementary Figures S1c and d). Since HPSE is a secreted enzyme, we also examined HPSE secretion in conditioned media (CM) by ELISA in NEK2 OE and EV ARP1 and OCI-MY5 MM cell lines; soluble HPSE levels were significantly increased in NEK2 OE MM cells compared to control MM cells (Supplementary Figure S1e; $P<0.05$ ).

To determine whether high NEK2 promotes osteoclast (OCL) differentiation, bone marrow macrophages (BMMs) derived from primary MM samples were co-cultured for up to 14 days with $50 \%$ conditioned media (CM) from NEK2 OE and control (EV) MM cell lines, ARP1 and OCI-MY5. Details are in Supplementary Methods. Tartrate-resistant acid phosphatase (TRAP) staining was performed to evaluate full $\mathrm{OCL}$ differentiation. $\mathrm{TRAP}^{+} \mathrm{OCLs}$ were not detected in BMMs only incubated with regular media (data no shown). The number of $\mathrm{TRAP}^{+} \mathrm{OCL}$ was significantly increased after co-culturing with NEK2 OE CM compared to EV CM (Figure 2a; $P<0.05$ ). Consistently, the number of TRAP ${ }^{+} \mathrm{OCL}$ was significantly decreased after co-culturing with NEK2 shRNA CM (Figure 2a; $P<0.05$ ) when compared to EV CM. Osteoclast quantification was determined in each sample (Supplementary Figure S2a). We subsequently examined whether HPSE was involved in NEK2-induced osteoclastogenesis in NEK2 OE MM cells. A doxycycline-inducible HPSE shRNA (NEK2 OE-shHPSE) or scrambled control was transfected into NEK2 OE ARP1 and OCI-MY5 MM cells to determine whether knockdown of HPSE decreases NEK2-induced OCL differentiation. As shown in the Figure $2 b$ and Supplementary Figure $S 2 b$, the number of TRAP ${ }^{+}$OCLs was significantly decreased from $4.4 \pm 1.7$ and $5.3 \pm 2.5$ per area to $1.9 \pm 1.1$ in ARP1 and $1.4 \pm 1.1$ in OCl-MY5 cell lines, respectively (Supplementary Figure S2b; $P<0.05$ ). We also tested the HPSE inhibitor, Roneparstat (formerly known as SST0001), in the culture media for $48 \mathrm{~h}, \mathrm{OCL}$ differentiation was significantly inhibited in NEK2 OE CM (data not shown). Consistently, OCL differentiation markers, such RANK, TRAP, NFATC1 and CTSK, were upregulated in BMMs cultured with NEK2 OE CM but this upregulation was abrogated by Roneparstat (OE SST) in both CMs collected from OCI-MY5 and ARP1 NEK2 OE cells (data not shown). These results support the conclusion that NEK2 induces $\mathrm{OCL}$ differentiation via upregulation and secretion of HPSE by MM cells.

We also confirmed that NEK2 is a driver of osteolysis in MM in vivo. ARP1 cells transfected with luciferase and NEK2 OE or EV were injected into NOD.CY-Rag1 mice via the tail vein $(n=6$ for NEK2 OE and $n=3$ for EV group). Details are in Supplementary Methods. One week after injection of MM cells, 3 of 6 NEK2 OE mice were treated with saline solution or HPSE inhibitor, Roneparstat $(60 \mathrm{mg} / \mathrm{kg}$, intraperitoneal injection/twice a day) for 3 weeks. All mice were killed when hind limb paralysis occurred in the control 
group mice. Human kappa light chain secretion was analyzed as an indirect indicator of tumor burden (data not shown). The femur and tibia bones from one mouse leg were fixed with $10 \%$ neutral buffered formalin for 7 days for the analyses by X-ray and microCT. The femur and tibia bones from another leg were decalcified for 2 weeks for histological staining. X-ray radiology analysis revealed bone lytic lesions in the hind limb of mice injected with NEK2 OE cells (Figure $2 \mathrm{c}$ ). MicroCT showed that bone volume and thickness of trabecular bone were decreased significantly in mice injected with NEK2 OE ARP1 cells compared to EV ARP1 cells. Strikingly, treatment with the HPSE inhibitor Roneparstat (NEK OE SST) markedly reduced bone mass loss for both trabecular bone and cortical bone thickness induced by NEK2 overexpression (Supplementary Figure S2c). TRAP staining of decalcified bone sections revealed a significant increase in the number of OCLs in the trabecular bone area in mice injected with NEK2 OE cells compared to EV mice, while addition of the Roneparstat reduced the number of OCLs (Supplementary Figure S2d).

The scope of this work was to determine the functional role of NEK2 in mediating bone destruction in MM and to apply this knowledge to develop novel therapies. In this study, we demonstrate that NEK2 plays an important role in MM bone disease. We evaluated the role of NEK2 in bone destruction of MM via clinical gene expression profiling data analysis and in vitro and in vivo mouse models. Further, treatment with the heparanase inhibitor Roneparstat, a Phase 1 drug in MM treatment, ${ }^{14}$ markedly inhibited $\mathrm{OCL}$ differentiation and rescued bone mass loss induced by NEK2 overexpression, suggesting that targeting NEK2 and/or its signaling pathway may be not only for prevent MM disease relapse but also bone destruction. Future study will explore the molecular mechanism(s) by which NEK2 upregulates and activates HPSE in MM cells resulting in bone damage in MM patients and determine whether NEK2 signaling is involved in osteoblast differentiation.

\section{CONFLICT OF INTEREST}

The authors declare no conflict of interest.

\section{ACKNOWLEDGEMENTS}

I thank Sigma-tau Research Switzerland (Mendriso, $\mathrm{CH}$ ) for providing the heparanase inhibitor Roneparstat. The data presented in this article were obtained at the Preclinical Imaging Shared Resource Facility, which is a Carver College of Medicine/ Holden Comprehensive Cancer Center core research facility at the University of lowa. The Facility is funded through user fees and the generous financial support of the Carver College of Medicine, Holden Comprehensive Cancer Center, and lowa City Veteran's Administration Medical Center. This work was supported by NIH grants R01CA152105 (FZ), R21CA143887 (FZ), R01CA138340 (RS), the Leukemia \& Lymphoma Society TRP (6094-12), the Multiple Myeloma Research Foundation (FZ), the International Myeloma Foundation (FZ), the American Society of Hemathology (ASH) Bridge (FZ), and institutional start-up funds from the Department of Internal Medicine, Carver College of Medicine, University of lowa (FZ and GT).

\section{AUTHOR CONTRIBUTIONS}

$\mathrm{MH}$ performed the experiments, collected, analyzed the data, generated the figures, wrote and editing the manuscript; RFM, HX performed the experiments; $J S, B B, D R, D E Q, S J, M H T, R D S$, LQ reviewed and edit the manuscript; IF, GT, FZ reviewed the data, wrote and edited the manuscript.

M Hao ${ }^{1,2}, \mathrm{R}$ Franqui-Machin ${ }^{2,3}, \mathrm{H} \mathrm{Xu}^{2}$, J Shaughnessy $\mathrm{Jr}^{4}$,

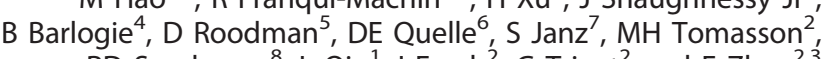
RD Sanderson ${ }^{8}$, L Qiu', I Frech ${ }^{2}, \mathrm{G}$ Tricot $^{2}$ and F Zhan ${ }^{2,3}$

${ }^{1}$ State Key Laboratory of Experimental Hematology, Institute of Hematology \& Blood Diseases Hospital, Chinese Academy of Medical Science \& Peking Union Medical College, Tianjin, China;
${ }^{2}$ Division of Hematology, Oncology, and Blood and Marrow Transplantation, Department of Internal Medicine, University of lowa, lowa City, IA, USA;

${ }^{3}$ Molecular \& Cellular Biology Program, University of lowa, lowa City, IA, USA;

${ }^{4}$ Department of Medicine, Mount Sinai School of Medicine, New York, NY, USA;

${ }^{5}$ Department of Medicine, Indiana University School of Medicine, Indianapolis, IN, USA;

${ }^{6}$ Department of Pharmacology, University of lowa, lowa City, IA, USA; ${ }^{7}$ Department of Pathology, The University of lowa Roy J and Lucille A Carver College of Medicine, lowa City, IA, USA and ${ }^{8}$ Department of Pathology, University of Alabama at Birmingham, Birmingham, AL, USA

E-mail: fenghuang-zhan@uiowa.edu or guido-tricot@uiowa.edu

\section{REFERENCES}

1 Kuehl WM, Bergsagel PL. Multiple myeloma: evolving genetic events and host interactions. Nat Rev Cancer 2002; 2: 175-187.

2 Billadeau D, Ahmann G, Greipp P, Van Ness B. The bone marrow of multiple myeloma patients contains $B$ cell populations at different stages of differentiation that are clonally related to the malignant plasma cell. J Exp Med 1993; 178: 1023-1031.

3 Zhou W, Yang Y, Xia J, Wang H, Salama ME, Xiong W et al. NEK2 induces drug resistance mainly through activation of efflux drug pumps and is associated with poor prognosis in myeloma and other cancers. Cancer Cell 2013; 23: 48-62.

4 Zhou W, Yang Y, Gu Z, Wang H, Xia J, Wu X et al. ALDH1 activity identifies tumorinitiating cells and links to chromosomal instability signatures in multiple myeloma. Leukemia 2014; 28: 1155-1158.

5 Faragher AJ, Fry AM. Nek2A kinase stimulates centrosome disjunction and is required for formation of bipolar mitotic spindles. Mol Biol Cell 2003; 14: 2876-2889.

6 Sonn S, Khang I, Kim K, Rhee K. Suppression of Nek2A in mouse early embryos confirms its requirement for chromosome segregation. Journal of Cell Science 2004; 117(Pt 23): 5557-5566.

7 Barlogie B, Tricot G, Anaissie E, Shaughnessy J, Rasmussen E, van Rhee F et al. Thalidomide and hematopoietic-cell transplantation for multiple myeloma. $N$ Engl J Med 2006; 354: 1021-1030.

8 Bartel TB, Haessler J, Brown TL, Shaughnessy Jr JD, van Rhee F, Anaissie E et al. F18-fluorodeoxyglucose positron emission tomography in the context of other imaging techniques and prognostic factors in multiple myeloma. Blood 2009; 114: 2068-2076.

9 Zhan F, Huang Y, Colla S, Stewart JP, Hanamura I, Gupta S et al. The molecular classification of multiple myeloma. Blood 2006; 108: 2020-2028.

10 Zhan F, Barlogie B, Arzoumanian V, Huang Y, Williams DR, Hollmig K et al. Gene-expression signature of benign monoclonal gammopathy evident in multiple myeloma is linked to good prognosis. Blood 2007; 109: 1692-1700.

11 Fux L, llan N, Sanderson RD, Vlodavsky I. Heparanase: busy at the cell surface. Trends Biochem Sci 2009; 34: 511-519.

12 Ramani VC, Zhan F, He J, Barbieri P, Noseda A, Tricot G et al. Targeting heparanase overcomes chemoresistance and diminishes relapse in myeloma. Oncotarget 2016; 7: 1598-1607.

13 Yang Y, Ren Y, Ramani VC, Nan L, Suva LJ, Sanderson RD. Heparanase enhances local and systemic osteolysis in multiple myeloma by upregulating the expression and secretion of RANKL. Cancer Res 2010; 70: 8329-8338.

14 Galli M, Magen H, Einsele H, Chatterjee M, Grasso M, Specchia G et al. Roneparstat (SST0001), an innovative heparanase (HPSE) inhibitor for multiple myeloma (MM) therapy: first in man study. Blood 2015; 126: 3246.

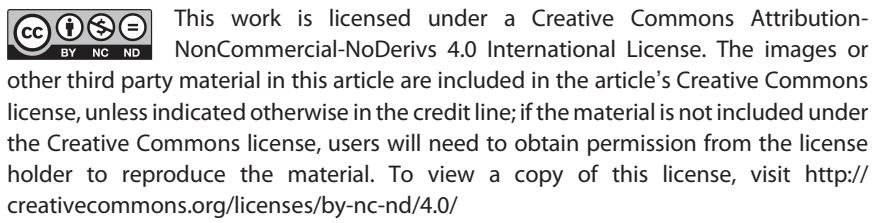

(c) The Author(s) 2017

Supplementary Information accompanies this paper on the Leukemia website (http://www.nature.com/leu) 